\title{
Traffic Analysis and Shaping of the Cloud Services over Common Deployment Model using Cloud Analyst
}

\author{
C.Saravanakumar \\ Research Scholar, Sathyabama University \\ Dept. of Computer Science and Engineering \\ Rajalakshmi Engineering College, Thandalam, \\ Chennai, Tamil Nadu, India
}

\author{
C.Arun \\ Associate Professor \\ Dept. of Information Technology \\ R.M.K College of Engg and Technology \\ Chennai, Tamil Nadu, India
}

\begin{abstract}
Cloud computing gives an enormous service to the customer in order to reduce the cost of investment on infrastructure development. The CSU (Cloud service User) can get all services from CSP (Cloud Service Provider) for satisfying their business needs. There are huge number of services are offered by the CSP for satisfying the customer's request. The performance of the cloud computing depends on various factors which provides the reliability over internet to the customer. One of the main factor need to be concentrates on reliability is network traffic because the service delay makes the customer dissatisfaction. The CSP may provide uneven traffic rate to CSU in order to manage the peak demand of their request. The fluctuation in the traffic may lead to the problem in retaining the customer i.e., the customer may feel difficult to access the service. This problem can be overcome by using the common deployment framework which gives the smooth traffic to the customer. The proposed work fully based on the traffic analyze and shaping the cloud services whenever the uneven traffic condition occurs. The traffic shaping has many features especially it is to handle the traffic rate. Cloud Analyst simulator can be used to analyze the cloud traffic with various parameters. The efficiency may be improved by introducing some new techniques on cloud service are the further work.
\end{abstract}

\section{Keywords}

Cloud computing, Interoperability, Cloud Analyst, cloud service.

\section{INTRODUCTION}

Cloud computing is the delivery of different computing service rather than resources, software, and information. It is directly used by the user. There are different types of services which satisfies the users request namely SaaS, PaaS, IaaS and etc,. Multi-tenancy is one of the features of the cloud computing is used to enable sharing of resources with the important characteristics namely centralization, peak load capacity and utilization with efficiency. The sharing of services in the cloud computing comprises the different layers such as Application Layer, Platform layer and Infrastructure layer. Different types of deployment model are used to provide the cloud services to the user namely Public Cloud, Community Cloud, Hybrid Cloud and Private Cloud. In a public cloud all services are accessed over the internet i.e., all the services are available globally. Community cloud shares infrastructure between several organizations from a specific community with common concerns whether it is managed internally or by a third-party and hosted internally or externally. The Hybrid cloud is a combination of public, community and private cloud in order to provide services to the users. Private clouds are providing the services within the organization which are managed internally. Large organizations needs to get the high quality services on mission-critical applications from the cloud service providers who does not offers guarantee of service availability, so the result can be a insufficient for mission-critical applications. This service is very suitable for small- and medium-sized organizations. There are many in-house IT services often fails to follow the standards which are not short out on the media scrutiny [1]. The amount of charges on cloud services provided by the cloud service providers are related to the usage of the storage as well as network bandwidth. A job is entering the cloud end which is processed by using the server, so the server needs to check the status. The static policies are not performed properly so it leads the server underutilized because of low traffic. The CSPs are facing lot of issues such as wasting energy, reducing the provider's revenues, overload during peak hours and missing profit opportunities. The existing approach suggests periodically invoking a resource allocation policy for estimating the traffic with server availability [2]. The complexity is more challenging while using private and public clouds. Cloud manufacturing is the process to provide effective solutions to the manufacturing industry for increasing customers with distributed manner. Cloud manufacturing is the way of conducting manufacturing businesses as s services and to provide the cloud customer. [3]. The UCA (Universal Connection Architecture) makes IA (Interactive Application) developers focus on the design without using unrelated knowledge and network APIs. This architecture can distribute the components over the networks without modifying the style or the source codes. The UCA has to extend for facilitating the deployment and the development of a cloud in order to handle the cloud services [4].The data integration system is used as a data flow style execution model, which is used to share the work and exploit the data by using the query evaluation [5]. In cloud computing the service requests are evaluated using the query evaluation because the requests are generated in the form of query. Session tracking, dynamic load-balancing, and persistence mechanisms are performed with Cloud SNAP architecture. The smooth 
transition may be achieved over small-scale to large-scale organization with the help of persistence interceptors [6]. The PROgram Ultra-Dispatcher (PROUD) allows people to configure by using their own computers or VMs in clouds are used to run applications rather than computers or virtual machines in clouds. The PROUD is used to regularize the rules for choosing run-time information such as CPU utilization, available memory, network activity, disk I/O activity, etc. It also balances the load across the customer and computer as well as VMs in the cloud with the help of load balanced policy [7]. In cloud computing, the nodes in media might be crash during transmission, so the protocol had to be introduced for solving these issues and also to achieve the error free transmission [8]. The accurate task and resource vector value can be achieved through learning in order to achieve the QoS of the cloud computing [9]. There are two types of heuristics for dynamic refinement namely clientbased and provider-based. Client-based heuristics can be used to adjust the configuration to improve the performance of individual classes where as Provider-based heuristics is for balancing the load and to improve system performance of the workload [10]. The cloud Analyst simulator is studying the large scale distributed applications and its behavior in the cloud environment. Global cloud service broker, which scale up and scale down the resources which are available in the application over different datacenter location [11]. The above works are not concentrating on some of the issues which are related to traffic management of the cloud services. The proposed work focuses on the network traffic optimization, shaping of bandwidth and traffic between the cloud customer end and service providers end. The proposed work also analyze the response time of the response which comes from various data centers on the cloud computing. Cloud Analyst is used for simulating and analyzing traffic for handling a cloud service with related parameters. The rest of the paper is organized as follows,

Section 1 represents the overview of cloud computing and related concepts. Section 2 describes a deployment framework of the proposed system; Section 3 represents the framework of traffic shaping of the proposed system. Section 4 represents the algorithm for bandwidth analyzing and selection. Section 5 represents architectural design of the proposed system. Section 6 represents simulation of traffic analysis using Cloud Analyst. Finally, Section 7 represents conclusion and future work.

\section{DEPLOYMENT FRAMEWORK OF THE PROPOSED SYSTEM}

Fig1.describes the common deployment framework of the cloud computing. The cloud customer requests the cloud services using the application programming interface. The common deployment layer has huge number of features which supports an efficient communication between customer and cloud provider. The deployment layer consists of service map-

\section{Application Programming Interface}

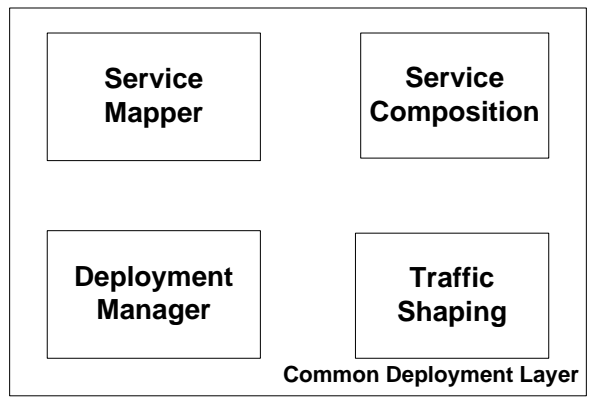

Cloud Service Providers

[Saas, PaaS, laaS. . . XaaS ]

Fig 1: Common Deployment Framework

per, service composition, deployment manager and traffic shaping. Service mapper maps the requests are coming from CSU using appropriate interface. The service composition has to composite the services by providing the quality of services. Deployment manager performs all the operations which are related to interoperability over cloud services. The traffic shaping is used to shape the traffic whenever the fluctuation occurs in the cloud communication. The correct traffic produced by the CSP to the valuable customer who often access more cloud services rather than basic customer. Suppose there are two customers request the cloud services one from company and other from individual user. The CSP may increase the bandwidth for the company's request because who use the cloud service long time when compared to individuals. Sometimes the user waits long time for required services because of low bandwidth. User may get unavailable services after spending the amount for the service's i.e. service unavailability. These problems can be overcome by managing the traffic with constant range for all the customers.

\section{FRAMEWORK FOR TRAFFIC SHAPING}

Fig . 2 describes the process flow of the Common Deployment Model. The local scheduler schedules the user requests and sent to the traffic scheduler. The traffic manager can able to manage the traffic within the level. It checks the traffic contract during the transfer. If the traffic level exceeds, an alert will be given to the user who knows the level of bandwidth of the current transfer. Traffic standard gives the policies and regulates the incoming and outgoing traffic of the cloud user's request. Traffic manager monitors the entire traffic and get the bandwidth limit of the current progress. Bandwidth selection selects the bandwidth and compared with the requested bandwidth using the matcher. If the requested bandwidth and current bandwidth are matched then only the services are sent to the cloud Service Providers for response. Monitor has to monitor and regulates all the CDM related activities for smooth process. The metering controller has to interact with the accounting and metering process for payment and gives the correct status of the current cost related parameters. 


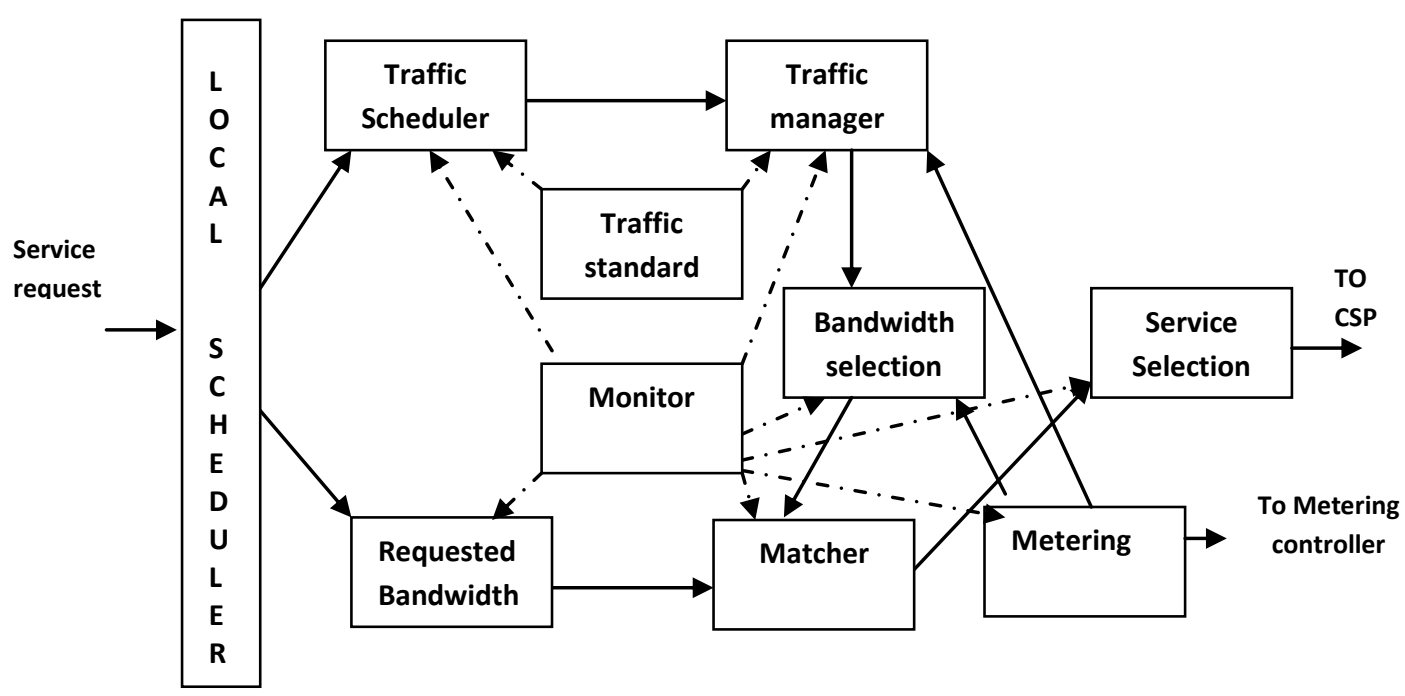

Fig 2: Traffic shaping of cloud services

\section{ALGORITHM FOR BANDWITH} ANALYZING AND SELECTION

\subsection{Bandwidth analysis and selection}

Bandwidth_analyzing_selection()

Input: cloud requests from the cloud service users;

Output: cloud service response from the cloud begin service providers;

Let CONN is the connection status, AN and DN is active node and dead node respectively;

Let BWAN is the bandwidth of active node;

Let CReqBW, CResBW are cloud request bandwidth and cloud response bandwidth

Let node_li;st $=\left\{\mathrm{n}_{1}, \mathrm{n}_{2} \ldots \mathrm{n}_{\mathrm{m}}\right\}$; respectively;

Local scheduler schedules the cloud request at the first node $\mathrm{n}_{1}$ i.e., starting node of the cloud request;

repeat

for each node $\mathrm{k}=1 \ldots \mathrm{n}_{\mathrm{m}} \quad$ do

find out the connection status of all the nodes as CONN;

for each conn $€$ CONN do

if conn $==$ connected then add the node in active_node AN; else

add the node in dead_node DN; end

end

end

for each active_node $€$ AN do

find out the bandwidth of active node as

BWAN;

end

Calculate the bandwidth for the requesting cloud services CReqBW.

Select a suitable bandwidth from BWAN;

Requests are sent to the cloud providers for

cloud service;

Cloud provider select suitable services and send to the cloud customer;

Calculate the response bandwidth from cloud provider as CResBW; for each

bandwidth_req $€ \mathrm{CReqBW}$ do

for each bandwidth_res $€$ CResBW do

if (bandwidth_req $==$ bandwidth_res) then

Send the service response to the cloud user;

else if (bandwidth_res < bandwidth_req) then

Send the alert signal to the cloud customer for unnecessary waiting and also send the query for the bandwidth deviation; Cloud provider can shape the traffic for send the

$$
\begin{aligned}
& \text { end } \\
& \text { end }
\end{aligned}
$$

until stopping condition is true;

end;

\subsection{Traffic shaping}

Traffic_Shaping ()

begin

Input: cloud service request with suitable traffic rate;

Output: cloud service response with suitable traffic rate;

Let Cloud Service Cost be a CSC;

Let Cloud Error Rate be a CER;

Let Cloud Service Rate be a CSR;

Let Available Bandwidth be an AB;

Let Response Time be a RT;

Let Number request is $\mathrm{N}$;

For each $\mathrm{n}$ in $\mathrm{N}$ do begin

L1: Select the bandwidth BW from AB; if $((\mathrm{BW}$. req $==\mathrm{AB}$.size $)$ and $(\mathrm{BW}$. req $<$

AB.size )) then 
goto L2;

else if (BW.req > AB.size) then

\{

Wait for Required BW for further process; goto L1; \}

L2: Processing the request at cloud service provider end;

Getting the cloud service response;

Find out the response rate:

Calculate the bandwidth response as BW. res; if $((B W \cdot$ res $==A B \cdot$ size $)$ and $(B W \cdot$.res $<$

\section{AB.size)) then}

Find out the Cloud Error Rate CER ;

if(!CER.value) then

\{

Success_rate++;

else

Failure_rate++;

If (Success_rate ) then

Calculate the CSC and RT for the cloud service response;

else

Stop the metering process temporarily;

Generate the query for failure and traffic delay;

The cloud service provider regularizes the traffic for query;

Resume the metering process for response;

Sending the response to the customer without delay and traffic problem;

$\mathrm{n}++$

end

end

\section{QOS OF THE CLOUD SERVICE}

Quality of the cloud service depends upon the QoS parameters like throughput, transit delay, error rates, priority, security, packet loss etc. the throughput is nothing but a bandwidth which are used to send and receive the service request as well as service response. The transit delay is defined as the time gap between sending request and receiving the service response. Number of errors occur during the transfer is determine by the error rate. The cloud service can be simulated with the help of Cloud Analyst and it also analyzes the QoS parameters which are describe in the following section.

\section{SIMULATION OF TRAFFIC}

\section{ANALYSIS USING CLOUD ANALYST}

The simulation mainly concentrates on traffic analysis with various parameter perspectives in order to maintain a smooth information flow between the cloud service request and cloud service response.

\subsection{Cloud Service Cost calculation}

The cost of the cloud service can be identified by using the following equation and also it is analyzed various data centers with the cost of core parameters such as VM, Memory etc., which gives us the complete response of cloud service request. Fig 3. describes the comparative study of different data centers with the service cost.

Cloud service cost $=(\text { (number of requests per user })^{*}$

( data size per request $)+$ ( starting of peak hours - ending of peak hours $)) /($ on peak service rate - off peak service rate )

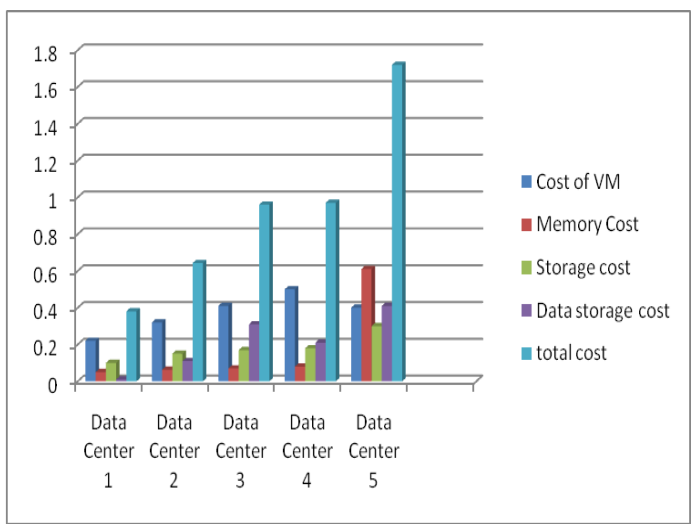

Fig 3: Comparison of Cloud Service Cost

\subsection{Comparison of cloud error rate}

The cloud service error rate can be calculated by using the following equation which gives the comparison of various cloud service errors in fig 4.

Cloud error rate $=($ number of request - number of response) $* 100$

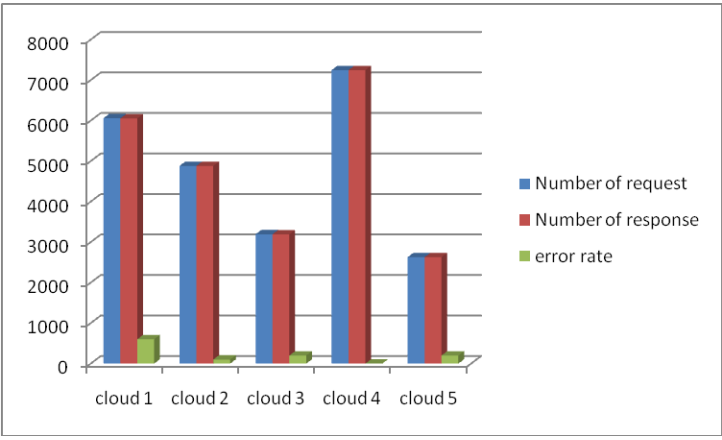

Fig 4: Comparison of cloud error rate

\subsection{Comparison of cloud response rate}

The cloud request is sent to the cloud service providers for getting the available service with the proper response can be measured based on the bandwith of the request as well as response described in fig 5 .

Response rate $=($ CresBW / 1024 $) * 1000$ 


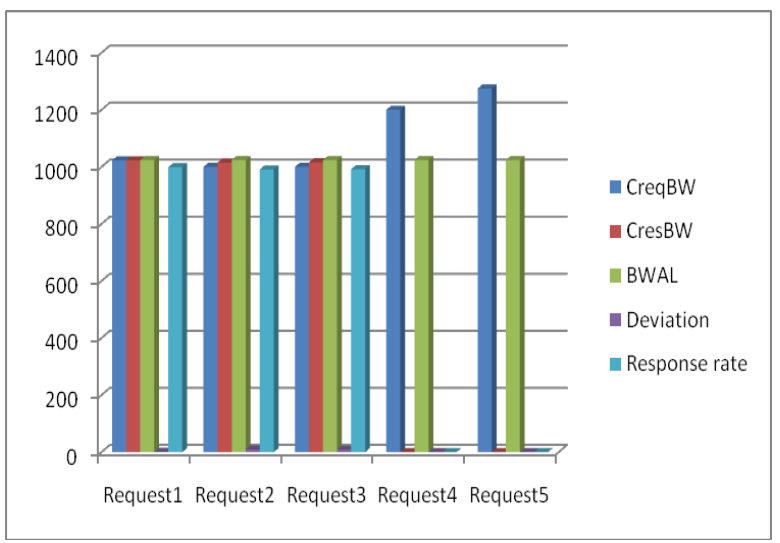

Fig 5: Comparison of cloud response rate

\section{CONCLUSION}

Large number of services is exchanged between the cloud service users and cloud service providers in order to achieve the expectation in both the ends. The cloud customer generates the request for accessing the cloud services from the cloud providers with high quality response. Cloud service provider may give the uneven traffic rate to the customer with their service access level. Normal user uses the cloud service in a short duration, when compared to other expensive customer. So the result is that the user can gets the traffic in different level which means that the normal user may get service unavailability even though he pay for the unavailable services. In order to overcome this problem the common deployment framework has introduced for analyzing and shaping the traffic for smooth information flow. This model consists of traffic shaping which monitors and control the traffic for accessing the cloud service. The Cloud Analyst simulator is used for analyzing and shaping of the traffic and also identified various parameters in order to impose quality in the cloud access. The QoS can be analyzed and based on various parameters of the cloud services for achieving the efficient transfer of information. The efficiency may be improved by introducing some new techniques on cloud service are the further work.

\section{REFERENCES}

[1] Sean Marston, Zhi Li, Subhajyoti Bandyopadhyay, Juheng Zhang , Anand Ghalsasi " Cloud computing The business perspective ", page no 176-189.

[2] Michele Mazzuccoa, Dmytro Dyachuk ,"Optimizing Cloud providers revenues via energy efficient server allocation", Institute of Computer Science, University of Tartu, Liivi 2, 50409 Tartu, Estonia page no 1-12.

[3] Xun $\mathrm{Xu}$, “From cloud computing to cloud manufacturing "page no $75-86$

[4] Tzu-Chi Huang, "Universal connection architecture for interactive applications to achieve distributed computing" page 232-251

[5] Gang Chen,YongweiWun, JiaLiu, GuangwenYang, Weimin Zheng," Optimizationo fsub-query processing in distributed data integration systems", page 1035 -1042

[6] Rubén Mondéjar, Pedro García-López, Carles Pairot, Lluis Pamies- Juarez" CloudSNAP: A transparent infrastructure for decentralized web deployment using distributed interception", page 1-11

[7] Tzu-Chi Huang, "Program Ultra-Dispatcher for launching applications in a customization manner on cloud computing",page 423- 446

[8] Shun-Sheng Wang, Kuo-Qin Yan *, Shu-Ching Wang**,"Achieving efficient agreement within a dualfailure cloud-computing environment “, page 906 - 915

[9] Baomin Xu , Chunyan Zhao , Enzhao Hua, Bin Hu,” Job scheduling algorithm based on Berger model in cloud environment", page 419 - 425

[10] Rizwan Miana,*, Patrick Martina, Jose Luis Vazquez poletti ,'Provisioning data analytic workloads in a cloud", page 1-19

[11] Rajkunar Buuya et al"Cloud Analyst: A Cloudsimbased visual Modeller for Analyuzing cloud computing environments and application", $24^{\text {th }}$ IEEE International Conference on Advanced Information Networking and Applications. page no 446-452

[12] S.Subashini et al., 2011, "A survey on security issues in service delivery models of loud computing ", Journal of Network and Computer Applications 34 pp. 1-11.

[13] John Harauz et al., $2009 \quad$ "Data security in the world of cloud computing", IEEE Computer and Reliability Societies, pp. 61- 64

[14] Balachandra Reddy Kandukrui et al, 2009, "Cloud Security Issues", IEEE International Conference on Services Computing, pp. 51-520.

[15] Gansan Zhao et al., 2010,"Deployment Models: Towards Eliminating Security Concerns from Cloud Computing", IEEE International Conference,, pp. 189 - 195 